\title{
Konflikte als Chance im Leitungshandeln Strategien zur Vermeidung und Bewältigung von Spannungssituationen
}

Der Umgang mit anderen Menschen, besonders in Führungssituationen, ist nie konfliktfrei. Insbesondere im Krankenhaus haben Konflikte zwischen den Berufsgruppen fast schon Tradition. Je heftiger sie auftreten, desto belastender sind sie für den Arbeitsalltag. Im schlimmsten Fall beeinflussen sie den Umgang mit den Patienten und damit die Patientenzufriedenheit und können so den Ruf der Klinik massiv beeinträchtigen und sogar wirtschaftliche Folgen haben.

In einer Fachabteilung eines Universitätsklinikums führte der Konflikt zwischen Chefarzt und Funktionspersonal und die damit verbundene Spaltung der Teams zunächst zu internen Beschwerden bei Personalrat, Vorstand und Aufsichtsrat bis hin zum Ministerium. Nachdem trotz Lösungsbemühungen keine Befriedung eintrat, kam es zu einer Eskalation in der lokalen und überregionalen Presse, die schließlich dazu führte, dass die Klinik kurz vor der Schließung stand.

\section{Was tun, wenn ein Konflikt auftritt?}

Tritt ein Konflikt auf, ist zunächst einmal zu klären, wie die Konfliktkonstellation aussieht, d.h. wie die Konfliktparteien gelagert sind. Haben Mitarbeiter untereinander Probleme, so ist die Führungskraft für die Klärung zuständig - was nicht bedeutet, dass sie die eigentliche Klärung herbeiführen soll oder muss. Ist der Chefarzt wie in unserem Fall jedoch in den Konflikt einbezogen, dann handelt es sich um einen „WIR-Konflikt“ und die Führungskraft sollte überlegen, ob sie einen professionellen Konfliktmoderator zur Konfliktlösung einladen möchte.

Zur Bearbeitung und damit zur Lösung eines Konfliktes ist es wichtig, diesen frühzeitig zu erkennen und geeignete Handlungen zur Lösung zu entwickeln, um eine derartige Eskalation und damit möglichen größeren Schaden zu verhindern. Konflikte zeigen sich häufig nur durch Symptome. Die beobachtbare Verhaltensweise der Konfliktpartner lässt Rückschlüsse auf den schwelenden Konflikt zu:

- Der Einzelne engagiert sich nicht in der gemeinsamen Arbeit

- Argumente werden mit großer Heftigkeit vorgetragen
- Mitglieder einer Gruppe gehen ungeduldig miteinander um

- Gruppenmitglieder sind nicht mehr bereit, aufeinander einzugehen

- Die Beteiligten äußern Zweifel am Sinn der Handlungen der jeweils anderen und wirken oder sind frustriert

- Es sind wechselseitig subtile persönliche Angriffe oder Spitzen erkennbar

In solchen Fällen lautet der erste Ansatz: „Konflikt auf den Tisch und Methoden suchen, diesen zu bearbeiten". Dabei ist es nicht immer einfach, die geeignete Methode zu finden. Gerade bei Konflikten, die sich über einen längeren Zeitraum erstrecken, sind die Fronten zunehmend verhärtet und eine offene, wertschätzende Kommunikation über die Konfliktthemen nahezu unmöglich.

\section{Konflikte ansprechen ohne Konfrontieren}

Eine geeignete Möglichkeit, Konflikte zu thematisieren, ohne dabei das Gegenüber zu konfrontieren, bieten Verfahren des „indirekten Dialogs”. Zielsetzung dabei ist, die Dialogfähigkeit zwischen den Konfliktparteien wiederherzustellen und gleichzeitig die Ängste der Mitarbeiter vor einer direkten Konfrontation zu mindern.

Die Konfliktbearbeitung erfolgt in 3 moderierten Sitzungen, die in den Konfliktparteien - ebenfalls unter moderierter Anleitung - vorbereitet werden.

\section{Der Wunsch}

Im Rahmen der ersten vorbereitenden Sitzung formulieren die Konfliktparteien bzw. Berufsgruppen ihre Anliegen an die jeweils andere Berufsgruppe. Bei der Formulierung der Wünsche wird zunächst die konkrete Beobachtung und eigene Wahrnehmung geschildert und das Problem in Daten und Fakten beschrieben: Was beobachten wir? Was nehmen wir mit unseren Sinnen wahr, entweder bei uns oder beim Gegenüber? Wichtig hier- 
bei ist, dass die Beobachtung ohne Bewertung der Situation geschildert wird. In einem zweiten Schritt wird die Folge der Beobachtung reflektiert: wie kommt das Beobachtete bei uns an und welche Gefühle löst die Beobachtung in uns aus? Zuletzt erfolgt die Formulierung der Bitte zur Veränderung: was können wir bzw. was können die anderen tun, um unseren Bedürfnissen eher zu entsprechen? Worum möchten wir jetzt bitten?

Die in dieser Systematik erarbeiteten Wünsche werden in einer Gesamtrunde allen Konfliktparteien vorgestellt. Wesentlich für den weiteren Konfliktbearbeitungsprozess ist es, dass im Rahmen der Vorstellung der Wünsche diese weder kommentiert noch bewertet werden. Alle hören sich gegenseitig zu und gehen ohne Diskussion über die Inhalte wieder auseinander.

\section{Das Angebot}

Mit dem Gehörten gehen die Mitarbeiter wieder zurück in ihre Berufsgruppen bzw. Konfliktparteien und es folgt die Auseinandersetzung mit den an sie selbst herangetragenen Anliegen. In einer moderierten Sitzung formulieren die jeweiligen Gruppen nun das Angebot, welches sie auf die Wünsche unterbreiten können. Dabei ist es unerlässlich, sich in die Situation des anderen hineinzuversetzen und das beschriebene Gefühl ernst zu nehmen.

Im Rahmen einer zweiten Gesamtrunde werden die Angebote aller Konfliktparteien offen ausgesprochen. Auch hier gilt: alle hören sich gegenseitig zu. Die Angebote werden weder kommentiert noch bewertet.

\section{Die Annahme}

Der letzte Prozessschritt in der Konfliktbearbeitung bildet die Angebotsannahme, die den Schulterschluss der Konfliktparteien herbeiführt. Im Rahmen einer moderierten Sitzung setzen sich die Teams mit den auf ihre Anliegen entgegengebrachten Angeboten auseinander und entscheiden, ob diese tragbar sind und den von ihnen geäußerten Bedürfnissen entsprechen. Hierbei ist wichtig, genau zu prüfen, ob das Angebot ausreicht, den entstandenen negativen Gefühlen zu begegnen oder ob ein ergänzendes Angebot notwendig ist. In der letzten Gesamtrunde der Konfliktparteien wird das Ergebnis vorgestellt und die geschlossene Vereinbarung fixiert.

\section{Faktoren zum Gelingen des Konfliktbearbeitungsprozesses}

Entscheidend für das Gelingen des Konfliktbearbeitungsprozesses sind folgende Faktoren, die zu Beginn gegenüber allen Beteiligten klar ausgesprochen werden sollten:

\section{Die Führungskraft als Vorbild}

Mitarbeiter orientieren ihr Verhalten am Verhalten ihrer Vorgesetzten. Gerade in Konfliktbearbeitungsprozessen, bei denen Führungskräfte am Konflikt beteiligt sind, ist es daher unerlässlich, dass die Führungsriege den Prozess geschlossen unterstützt und als Vorbild voranschreitet. Dazu gehört vor allem, sich durch wertschätzende Kommunikation und unbelastetes Zuhören an den Gesamtrunden zu beteiligen.

\section{Die Kunst des Zuhörens}

Aktives Zuhören bedeutet, sich in sein Gegenüber einzufühlen und für eine be- grenzte Zeit einen Perspektivenwechsel vorzunehmen, d.h., die Welt aus den Augen des anderen zu sehen. Damit dies gelingen kann, ist es ganz besonders wichtig, sich nicht von eigenen Assoziationen und Bewertungen ablenken $\mathrm{zu}$ lassen, sondern so zuzuhören, als müsste man das Gesagte anschließend uneingefärbt wiederholen.

Die wertschätzende Kommunikation In Konfliktsituationen sind Menschen dazu geneigt, problemorientiert und verallgemeinernd zu formulieren und gegenüber ihrem Konfliktpartner in eine Vorwurfshaltung zu gehen. Gerade dann ist eine wertschätzende Kommunikation allerdings unbedingt notwendig, um Konfrontation, Aggression und letztendlich eine Verhärtung des Konflikts zu vermeiden. Die oben beschriebene Systematik zur Thematisierung von Problemen unterstützt eine wertschätzende Formulierung der Anliegen. Die Vermittlung der eigenen Wahrnehmung in Form von Ich-Botschaften anstelle von vorwurfsbehafteten DuBotschaften steht dabei im Vordergrund.

\section{Der objektive Konfliktmoderator}

Ist die Führungskraft am Konflikt beteiligt, wird eine Doppelrolle Führungskraft und Konfliktmoderator nicht gelingen und ein externer, neutraler Konfliktmoderator oder Mediator ist unerlässlich, um den Konfliktbearbeitungsprozess erfolgreich zu steuern. Ziel des Konfliktmoderators ist dabei nicht, den Konflikt zu lösen, sondern Spielregeln für den Konfliktbearbeitungsprozess zu etablieren und den Rahmen für ein künftiges konfliktfreies Miteinander abzustecken.

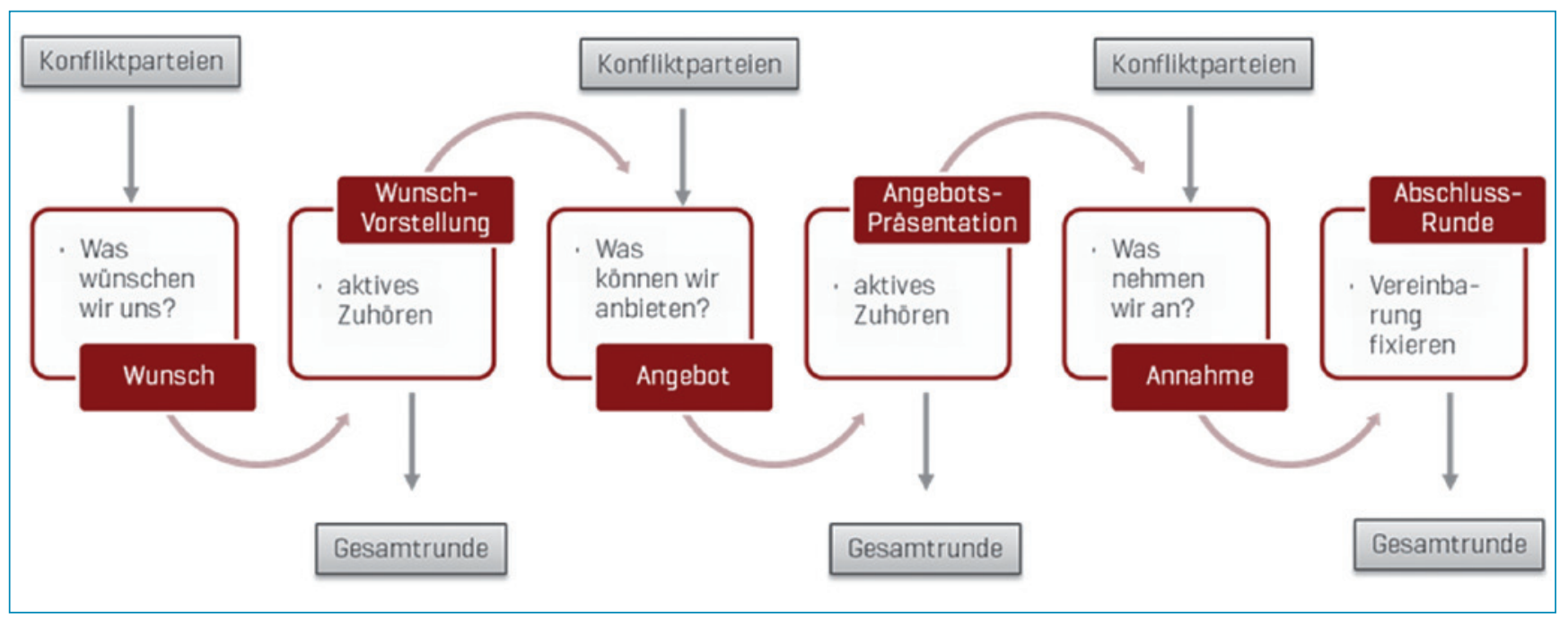

Abb. 1 Verfahren des ,indirekten Dialogs“. 


\section{Die Einigung durch Prozesse stabilisieren}

Konflikte müssen nicht immer zwischenmenschliche Ursachen haben. Auch in unserem Projektbeispiel lagen einige der Ursachen in ineffizienten Arbeitsabläufen, fehlenden Zuständigkeiten oder mangelnder Verantwortungsübernahme. Um die getroffenen Vereinbarungen zu stabilisieren, empfiehlt es sich daher, unterstützende Prozesse zu etablieren, die das Risiko eines Wiederauftretens der Konflikte verringern und den Mitarbeitern auf Arbeitsebene Sicherheit bieten. Welche Prozesse hierbei unterstützen, ist abhängig vom jeweiligen Konflikt. In unserem Fall entzündete sich ein Teil der Spannungen zwischen den Berufsgruppen am Thema Fehlerkultur und mangelnder Wertschätzung in der Kommunikation. Die Einführung eines Prozesses für Fehlermanagement sowie die regelmäßige Durchführung von Mitarbeiterentwicklungs- und Feedbackgesprächen boten sich an, um solchen Konflikten auf der Prozessebene zu begegnen und eine Verlagerung des Problems auf die emotionale zwischenmenschliche Ebene zu verhindern.

Weitere Prozessoptimierungen wurden an Schnittstellen (z.B. Abstimmungswege zwischen Ambulanz, Diagnostik und Station) sowie in der Aufbauorganisation durch Abbau einer Leitungsebene vorgenommen.

Grundsätzlich positiv wirkte die Erarbeitung eines Führungsstatements, in welchem die Unternehmenswerte sowie die strategischen Stoßrichtungen durch die Führungsmannschaft schriftlich dargelegt wurden. Insbesondere in Veränderungsprozessen, die mit der Bearbeitung eines Konfliktes automatisch einhergehen, bietet ein solches Führungsstatement Orientierung, Perspektive und Motivation und richtet den Blick auf die künftig zu lösenden Sachthemen.

\section{Die Berufsgruppen für künftige Konflikte wappnen}

Die Vermeidung künftiger Konflikte ist nach Auflösung bestehender Konflikte ein zentrales Thema. Die Mitarbeiter benötigen Methoden und Instrumente an die Hand, die einen wertschätzenden Umgang miteinander fördern und bei bevorstehenden Konflikten korrigierend greifen. Folgende Möglichkeiten sind je nach Konfliktkonstellation hilfreiche Unterstützer für das künftige Zusammenarbeiten:

\section{Einzelcoaching}

Entzündet sich der Konflikt schwerpunktmäßig an einzelnen Personen, so ist ein Einzelcoaching für den jeweiligen Mitarbeiter ein effektives Instrument, um seine innere Haltung zu reflektieren. Dabei geht es insbesondere darum, in persönlichen zwischenmenschlichen Konflikten die Andersartigkeit des Konfliktpartners nicht als Abgrenzung, sondern als Ergänzung wahrzunehmen und damit die häufig daraus resultierende Abwertung und mangelnde Wertschätzung in Respekt umzukehren.

\section{Kommunikationstrainings}

Missverständnisse in der Kommunikation sind häufige Auslöser für Konflikte, z.B. wenn das Senden und Entschlüsseln von Botschaften auf unterschiedlichen Ebenen erfolgt, die Kommunikation tendenziell problem- statt lösungsorientiert ist oder es an Strategien für den Umgang in schwierigen Gesprächssituationen (z.B. bei kritischem Feedback) fehlt. Solche Missverständnisse sind durch Sensibilisierung des individuellen Kommunikationsverhaltens leicht zu umgehen.

Das Vier-Ohren-Modell oder das Wertequadrat nach Friedemann Schulz von Thun sind leicht zugängliche Modelle, die im Rahmen eines Kommunikationstrainings vermittelt und in der Umsetzung im täglichen Berufsalltag begleitet werden können.

\section{Teamentwicklung}

Die Intelligenz eines Teams überschreitet die Intelligenz des Einzelnen bei Weitem. Ein Team kann außergewöhnliche Fähigkeiten zum koordinierten Handeln entwickeln. Dafür müssen die Teammitglieder jedoch eigene Annahmen aufheben und sich auf ein echtes gemeinsames Denken einlassen. Dazu gehört auch, dass ein Team bestimmte Interaktionsstrukturen erkennt und z.B. gegenseitige Abgrenzungs- oder Abwehrmechanismen abbaut, sodass die vielseitigen Perspektiven und die unterschiedlichen Expertisen erfolgreich eingebracht werden können. Im Rahmen von Teamentwicklungsmaßnahmen wird bei den Mitarbeitern die Grundlage geschaffen, um erfolgreich Gruppen- und Teamprozesse zu gestalten und Konflikte zu vermeiden.

\section{Fazit}

Das Konfliktpotential am Arbeitsplatz nimmt stetig zu. Die rasante Entwicklung

der Märkte und die damit einhergehenden Veränderungsprozesse in den Unternehmen sowie der zunehmende Arbeitsund Zeitdruck hemmen die Motivation der Mitarbeiter und erschweren ein zielorientiertes, gemeinsames Arbeiten. Gerade im Krankenhaus, wo der Umgang mit teils schwerkranken Patienten zu extremer psychischer Belastung der Mitarbeiter führt, werden Konflikte sowohl zwischen als auch innerhalb der Berufsgruppen kaum zu vermeiden sein.

Umso wichtiger ist es für die Führungskraft, für den Konfliktfall gut aufgestellt zu sein und über Strategien zur Konfliktvermeidung und Konfliktbewältigung zu verfügen. Die Kombination effektiver Konfliktmoderation mit flankierender Optimierung relevanter Prozesse war für die Klinik des Universitätsklinikums dabei ein erfolgreicher Weg, der zu einer Auflösung des Konfliktes bei gleichzeitig nachhaltiger Verbesserung der Strukturen und Zusammenarbeit im Team geführt hat.

\section{Korrespondenz}

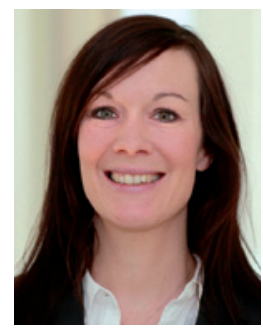

Katharina Ade

Ruhl Consulting AC Harrlachweg 1 68163 Mannheim Tel.: 0621/32886490

E-Mail: info@ruhl-consulting.de Internet: www.ruhl-consulting.de 